\title{
Reflets
}

Revue d'intervention sociale et communautaire

\section{Les pratiques émergentes de lutte contre l'homophobie dans les milieux scolaires et jeunesse de l'Outaouais}

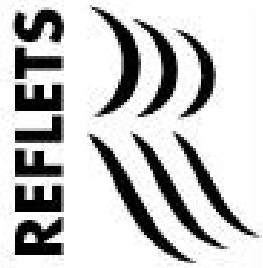

\section{Sylvie Thibault, Kévin Lavoie et Vincent Chouinard}

Volume 19, numéro 1, printemps 2013

Approches structurelles et intervention sociale

URI : https://id.erudit.org/iderudit/1018045ar

DOI : https://doi.org/10.7202/1018045ar

Aller au sommaire du numéro

Éditeur(s)

Reflets, Revue d'intervention sociale et communautaire

ISSN

1203-4576 (imprimé)

1712-8498 (numérique)

Découvrir la revue

Citer cet article

Thibault, S., Lavoie, K. \& Chouinard, V. (2013). Les pratiques émergentes de lutte contre l'homophobie dans les milieux scolaires et jeunesse de l'Outaouais. Reflets, 19(1), 153-184. https://doi.org/10.7202/1018045ar
Résumé de l'article

Cet article présente les résultats d'uneétude menée auprès de onze intervenants etenseignants de la région de l'Outaouais.Réalisée en Outaouais avec l'étroitecollaboration de Jeunesse Idem, service d'information et desoutien pour les jeunes (âgés de 14 à 25 ans) deminorités sexuelles, cette démarche exploratoire a permisde documenter les pratiques émergentes visant à contrerles violences homophobes auprès des jeunes.

Plusspécifiquement, le point de vue des répondants sur lesphénomènes de l'homophobie et del'hétérosexisme dans les milieux jeunesse estexploré. En outre, cet article expose les stratégies desensibilisation développées par les acteurs sociaux etqui sont établies par le biais de différentescollaborations entre les écoles et la communauté. Lesfacteurs facilitant de telles alliances sont identifiés, ainsique ceux qui inhibent l'émergence de pratiques de luttecontre l'homophobie. Des pistes de recherches futures et lesimplications pour la pratique sont également discutées.
Tous droits réservés @ Reflets, Revue d’intervention sociale et communautaire, 2013
Ce document est protégé par la loi sur le droit d'auteur. L'utilisation des services d'Érudit (y compris la reproduction) est assujettie à sa politique d'utilisation que vous pouvez consulter en ligne. 


\section{Les pratiques émergentes de lutte contre l'homophobie dans les milieux scolaires et jeunesse de l'Outaouais}

Sylvie Thibault, Ph. D.

Professeure et directrice, Département de travail social Université du Québec en Outaouais

Kévin Lavoie, B. Serv.Soc., t.s.

Étudiant à la maîtrise, Département de travail social

Université du Québec en Outaouais

Vincent Chouinard, M.Serv.Soc., t.s.

Travailleur social

Résumé

Cet article présente les résultats d'une étude menée auprès de onze intervenants et enseignants de la région de l'Outaouais. Réalisée en Outaouais avec l'étroite collaboration de Jeunesse Idem, service d'information et de soutien pour les jeunes (âgés de 14 à 25 ans) de minorités sexuelles, cette démarche exploratoire a permis de documenter les pratiques émergentes visant à contrer les violences homophobes auprès des jeunes. Plus spécifiquement, le point de vue des répondants sur les phénomènes de l'homophobie et de l'hétérosexisme dans les milieux jeunesse est exploré. En outre, cet article expose les stratégies de sensibilisation développées par les acteurs sociaux et qui sont établies par le biais de différentes collaborations entre les écoles et la communauté. Les facteurs facilitant de telles alliances sont identifiés, ainsi que ceux qui 
inhibent l'émergence de pratiques de lutte contre l'homophobie. Des pistes de recherches futures et les implications pour la pratique sont également discutées.

Mots clés : pratiques émergentes; homophobie; hétérosexisme; intervenants sociaux; enseignants

\section{Abstract}

This article presents the results of a study conducted with eleven social practitioners and teachers from the Outaouais region. This exploratory approach has documented emerging practices to counter homophobic violence among young people. More specifically, the views of respondents on the phenomena of homophobia and heterosexism is explored. Also, outreach strategies developed by social actors are described through various collaborations established between schools and the community. Facilitating factors such alliances are identified, as well as those which inhibit the emergence of practices against homophobia. Avenues for future research and implications for practice are discussed.

Key words : Emerging Practices; Homophobia;Heterosexism; Social Practitioners; Teachers

\section{Contexte de la recherche}

Au cours des vingt dernières années, plusieurs chercheurs et intervenants se sont intéressés aux questions liées à l'homophobie et à l'hétérosexisme chez les jeunes, notamment dans les milieux scolaires et jeunesse. Certaines de ces études ont documenté le point de vue des jeunes de minorités sexuelles (Bauermeister, 2010; Kosciw, et collab., 2010; Poteat, 2007). D’autres ont mesuré l'impact des expériences de victimisation vécues par ces jeunes sur leur cheminement scolaire (Chamberland, et collab., 2011; Osborne et Wagner, 2007; Tharinger, 2008; Walton, 2004) ou ont exploré les liens entre l'homophobie, l'hétérosexisme et le suicide (Dorais et Lajeunesse, 2000). En outre, quelques chercheurs se sont 
"...la violence

homophobe touche au secondaire près de quatre élèves sur dix..."

«...les programmes de prévention et de sensibilisation organisés y sont parfois peu accessibles ou méconnus..." intéressés aux pratiques de lutte contre l'homophobie (Benton, 2003; Goldstein, Collins et Halder, 2008; Graybill, et collab., 2009) principalement en documentant les divers programmes offerts dans les écoles secondaires ou les établissements postsecondaires (Buston et Hart, 2001; Chouinard, 2010; Schniedewind et Cathers, 2003; Swank, et collab., 2008; Valenti et Campbell, 2009; Walls, Kane et Hope, 2010).

Les études étasuniennes et canadiennes rapportent toutes que la situation atteint son paroxysme à l'école secondaire. De même, les résultats de l'enquête québécoise de Chamberland et collab. (2011) abondent dans le même sens et révèlent que la violence homophobe touche au secondaire près de quatre élèves sur dix $(38,6 \%)$, peu importe leur orientation sexuelle, réelle ou présumée.

Jusqu'à maintenant, peu d'études canadiennes se sont attardées aux pratiques sociales de lutte contre l'homophobie. La recension des écrits à ce sujet indique que les pratiques émergent souvent par nécessité des milieux scolaires et jeunesse, puisque les programmes de prévention et de sensibilisation organisés y sont parfois peu accessibles ou méconnus (Thibault, Chouinard et Lavoie, à paraitre). À la lumière de ces différents constats, on peut penser que l'absence, la rareté ou le manque de visibilité des ressources concernant la diversité sexuelle puissent conduire certains acteurs sociaux (enseignants, intervenants, travailleurs communautaires, etc.), impliqués dans les milieux jeunesse (écoles, maisons de jeunes, etc.), à mettre en place des initiatives originales dans le but de sensibiliser les jeunes ou de contrer les violences homophobes dans leur milieu. Ces pratiques ont comme principale caractéristique d'être développées in situ et sont la plupart du temps inconnues au-delà du milieu isolé duquel elles émergent. Pourtant, ces savoirs d'expériences pourraient répondre à des besoins dans d'autres milieux où des intervenants (enseignants, animateurs, intervenants sociaux) sont aux prises avec des situations liées à l'homophobie et aux conséquences qui lui sont associées. Le présent article vise précisément à présenter les résultats d'une étude réalisée auprès d'intervenants sociaux et d'enseignants de la région de l'Outaouais, laquelle avait pour 
but de documenter les pratiques de lutte contre l'homophobie auprès des jeunes.

\section{Problématique}

\section{Manifestations de l'hétérosexisme et de l'homophobie à l'école}

Les manifestations d'homophobie dans les milieux scolaires ont fait l'objet d'un nombre important d'études nord-américaines, mais aussi à l'échelle internationale (Richard, Chamberland et Petit, 2013). De façon générale, les auteurs dressent un portrait

"Les études recensées rapportent que ce sont les garçons beaucoup plus que les filles qui adoptent des attitudes et comportements homophobes, et que leurs gestes sont plus souvent dirigés envers les garçons plutôt qu'envers les filles." plutôt sombre de la situation. Les études recensées rapportent que ce sont les garçons beaucoup plus que les filles qui adoptent des attitudes et comportements homophobes, et que leurs gestes sont plus souvent dirigés envers les garçons plutôt qu'envers les filles (Buston et Hart, 2001; Chamberland, et collab., 2010; Grenier, 2005; Herek, 1988; Kimmel et Mahler, 2003; Osborne et Wagner III, 2007; Poteat et Espelage, 2005; Roper et Halloran, 2007).Au Québec, les résultats de Chamberland et collab. (2010) démontrent que des garçons plus que des filles ont posé des gestes de violence envers des élèves qui s'identifient comme lesbiennes, gais ou bisexuels (LGB), tels qu'insulter, se moquer, humilier (31,8\% contre 16,6\%); exclure ou rejeter $(17,6 \%$ contre $10,2 \%)$; bousculer, frapper, cracher dessus ou lancer des objets $(9,4 \%$ contre 3,3\%). Les auteurs soulignent que 74,3\% des jeunes ayant participé à leur enquête ont été témoins ou ont entendu parler d'une manifestation homophobe quelconque. Ces manifestations comprennent le fait d'insulter et d'humilier $(66,4 \%)$; de lancer des rumeurs $(58,8 \%)$; d'exclure et de rejeter (54,9\%); de faire de la cyberintimidation $(35,4 \%)$; de poser des gestes de violence physique (32,7\%); de vandaliser ou de voler (24,2\%). Les auteurs rapportent que plus de $57,9 \%$ des filles et $68,4 \%$ des garçons interrogés déclarent entendre souvent des remarques comme "c'est tapette ", " c'est fif " ou " c'est gai ", et c'est 40,5\% des garçons et $28,4 \%$ des filles qui soutiennent être souvent la cible d'insultes comme « fif ", « tapette ", « lesbienne " ou " homo " 
(Chamberland, et collab., 2010). Ces résultats exposent sans ambiguité que non seulement les jeunes LGB sont plus vulnérables à la victimisation homophobe que leurs pairs hétérosexuels (69\% contre $35,2 \%$ ), mais qu'ils sont jusqu'à trois fois plus à risque d'en vivre fréquemment et selon les diverses déclinaisons énumérées ci-dessus. En outre, ces résultats sont comparables aux taux recensés dans des études similaires (Émond et Bastien Charlebois, 2007). D'autres travaux réalisés en milieu scolaire démontrent que la présomption d'hétérosexualité y est souvent tenue pour acquise, que les manifestations de comportements homophobes sont considérées comme un stade "naturel " du développement des garçons et que certains professeurs sont parfois silencieux devant l'intimidation que certains jeunes subissent (Douglas, et collab., 1997; Epstein et Johnson, 1994; 1998; Mac an Ghaill, 1991; Nayak et Kehily, 1996; Rivers, 1996, dans Buston et Hart, 2001; Maher, et collab., 2009). Les études québécoises disponibles abondent dans le même sens.

\section{Impacts de l'homophobie et de l'hétérosexisme}

"Les effets négatifs de l'homophobie et de l'hétérosexisme sur la santé mentale des jeunes qui les subissent en milieu scolaire sont de mieux en mieux connus..."
Les effets négatifs de l'homophobie et de l'hétérosexisme sur la santé mentale des jeunes qui les subissent en milieu scolaire sont de mieux en mieux connus (Galliher, Rosotsky et Hugues, 2004; Richard, Chamberland et Petit, 2013; Saewyc, 2011). Les recherches répertoriées révèlent que les élèves victimes de cette violence sont plus à risque de manquer des cours, d'abandonner l'école, de tenter de s'enlever la vie, de fuguer ou de se prostituer, d'avoir des pratiques sexuelles à risque, de consommer de l'alcool et des drogues, de vivre de l'homophobie intériorisée ou de manquer d'estime de soi (Commission des droits de la personne et des droits de la jeunesse, 2007; Conseil permanent de la jeunesse, 2007; Émond et Bastien Charlebois, 2007; Maher et collab., 2009; Ryan, 2003; Taylor, et collab., 2010). Combiné au fait que les jeunes LGB reçoivent peu ou pas d'information sur les façons dont s'exprime leur sexualité, on peut comprendre qu'ils souffrent davantage que leurs pairs hétérosexuels de difficultés psychosociales liées au manque de reconnaissance de leur orientation sexuelle, à l'intimidation ou aux agressions dont ils sont victimes (Frappier, 
"...ils souffrent

davantage que leurs

pairs hétérosexuels de

difficultés psychosociales

liées au manque de

reconnaissance de leur

orientation sexuelle, à

l'intimidation ou aux

agressions dont ils sont

victimes. " et collab., 1997, dans Demczuk, et collab., 2003). En outre, on mentionne rarement l'impact que la honte, la haine de soi et la peur ont sur le cheminement sexuel et affectif de ces jeunes. Ces derniers vont parfois compartimenter leur vie ou s'imposer de vivre des relations hétérosexuelles qui "loin d'être de simples expérimentations et vérifications, sont un effacement de soi " (Émond et Bastien Charlebois, 2007, p. 51). Par ailleurs, les auteurs soulignent que "l'invisibilité, la présomption d'hétérosexualité, l'infériorisation de l'homosexualité, l'homophobie ambiante, le rejet réel ou appréhendé des jeunes LGB et l'absence ou l'inadéquation du soutien ont des impacts certains sur les jeunes " (Émond et Bastien Charlebois, 2007, p. 47).

\section{Le point de vue des jeunes}

"Les élèves interrogés sur l'homosexualité et l'homophobie soutiennent que l'on parle bien peu de l'homosexualité à l'école."
Les élèves interrogés sur l'homosexualité et l'homophobie soutiennent que l'on parle bien peu de l'homosexualité à l'école (Conseil permanent de la jeunesse, 2007). Cette absence du thème de l'homosexualité pourrait être en partie responsable de la perception souvent négative que s'en font les jeunes, toute orientation sexuelle confondue. Même si les résultats sont modulés selon le sexe du répondant (Émond et Bastien Charlebois, 2007), de façon générale, l'image de la lesbienne est moins péjorative que celle de l'homme gai qui est décrite de façon plus stéréotypée (Chambers, Van Loon et Tincknell, 2009; Émond et Bastien Charlebois, 2007). De plus, les élèves qui s'identifient comme lesbiennes, gais ou bisexuels reconnaissent que "les gais et les lesbiennes dont on ne devine pas l'orientation sexuelle ne seront pas stigmatisés [...] alors que les hétérosexuels dont les comportements laissent supposer une orientation homosexuelle risquent de vivre de l'intimidation "(Conseil permanent de la jeunesse, 2007, p. 24). Du reste, des élèves qui adoptent des comportements homophobes, une majorité ont l'impression d'être drôles (33\%) ou se sentent approuvés par les autres étudiants (25\%). Un peu moins de la moitié d'entre eux ressentent de la honte ou de la culpabilité $(46 \%)$ et seulement $6 \%$ de ces " agresseurs " disent avoir eu peur de se faire réprimander (Chamberland, et collab., 2010). De même, les témoins de ces comportements vont rarement 
dénoncer la violence. Lorsqu'interrogés, ils rapportent croire que la situation ne les regarde pas (39\%), que ce n'est pas assez important pour être dénoncé ( $23 \%)$, que rien ne sera fait (23\%), ou encore, par crainte des agresseurs, de devenir la cible de la violence ou de recevoir le même stigmate que la victime (Chamberland, et collab., 2010; Émond et Bastien Charlebois, 2007). Le portrait est assez semblable chez les victimes des manifestations homophobes, dont $51 \%$ croient que l'événement n'est pas assez majeur pour être dénoncé, $27 \%$ ont l'impression que rien ne sera fait pour corriger la situation, $15 \%$ craignent de passer pour des stooleurs (mouchards) et $15 \%$ appréhendent des conséquences négatives.

\section{L'attitude des enseignantes et des enseignants}

Les études ayant exploré l'attitude du personnel enseignant envers l'homosexualité dans les écoles secondaires démontrent de façon générale que celui-ci est animé par le désir de dispenser aux élèves une éducation sexuelle appropriée et non discriminante (Grenier, 2005; Martinez et Phillips, 2009). Toutefois, les auteurs répertorient des interventions plutôt sporadiques et ancrées dans le modèle biomédical, avec une tendance à lier sexualité et procréation dans un modèle hétérosexuel, et abordant les risques qui lui sont associés (grossesses, ITSS,VIH). Les élèves seraient dès lors considérés immatures et inaptes à recevoir de l'information qui mettrait l'accent sur le plaisir, l'autoérotisme et la diversité sexuelle au sens large (Buston et Hart, 2001; Martinez et Phillips, 2009; Richard, 2010). De fait, la majorité des enseignants et des intervenants scolaires interrogés sur leurs pratiques déplorent les lacunes de leur formation à ce propos (Conseil permanent de la jeunesse, 2007; Grenier, 2005; Maher, et collab., 2009; Martinez et Phillips, 2009; Richard, 2010). Les limites évoquées peuvent avoir comme conséquence de garder intactes chez certaines personnes enseignantes certaines conceptions de la diversité sexuelle ou faire en sorte qu'elles sous-estiment l'impact de l'humour à connotation homophobe (Buston et Hart, 2001). D'ailleurs, Grenier (2005) souligne que $34 \%$ des intervenants scolaires participant à son étude affirment avoir déjà fait de telles blagues. 


\section{Réponses sociales disponibles}

Quoique les jeunes lesbiennes, gais, bisexuels ou en questionnement (LGBQ) présentent les mêmes facteurs de risque que tous les adolescents, ils sont aux prises avec des défis supplémentaires liés à l'acceptation de leur orientation sexuelle (Elze, 2002). Certains auteurs ont démontré à quel point la réaction initiale des parents à l'annonce de l'orientation homosexuelle de leur enfant est une pierre angulaire dans le processus de coming out des jeunes LGB. Les auteurs soulignent l'importance accordée au soutien social et à l'existence de réseaux pour les jeunes lesbiennes, gais et bisexuels en situation de détresse et le rôle que peuvent aussi jouer le personnel enseignant ou les ressources professionnelles (Nesmith, Burton et Cosgrove, 1999). Les jeunes LGB qui subissent les manifestations d'homophobie se tournent le plus souvent vers leur famille ou leurs amis hétérosexuels. Dans une moindre mesure, certains vont chercher de l'aide auprès des ressources professionnelles ou du personnel enseignant, mais leur accessibilité ou leur disponibilité fluctuent (Conseil permanent de la jeunesse, 2007).

\section{Types d'initiatives et acteurs impliqués}

"Plusieurs initiatives ou projets de prévention et de lutte à l'homophobie sont créés et implantés en milieu scolaire par différents acteurs. "
Plusieurs initiatives ou projets de prévention et de lutte à l'homophobie sont créés et implantés en milieu scolaire par différents acteurs. Ils peuvent être mis en place par des enseignants (parfois en collaboration avec leurs étudiants), par la communauté, ou par les institutions publiques ou parapubliques. Ils peuvent avoir pour cible une classe, un ensemble de classes, voire toute l'école ou un regroupement d'écoles.

\section{Les initiatives du personnel enseignant}

Certains enseignants vont aborder les questions liées à la diversité sexuelle par des présentations, des discussions bonifiées par des visionnements de films, par des lectures obligatoires ou le font plus directement au gré de questions des étudiants ou à la suite d'une manifestation d'homophobie dont ils sont témoins (Conseil permanent de la jeunesse, 2007; Grenier, 2005; Richard, 2010). 
"Les associations d'enseignants, les regroupements professionnels ou les syndicats offrent aussi $d u$ soutien au personnel scolaire sous la forme de formations ou de partage de documentation et d'outils permettant le développement des compétences. "
Cependant, ces activités de sensibilisation et les discussions en classe sur le sujet demeurent rares (Chamberland, et collab., 2010).

Autre outil de prévention, les groupes appelés Gay-Straight Alliances (GSA) sont des associations dirigées par des étudiants et soutenues par le personnel scolaire. Elles ont pour but de faire la promotion de l'acceptation des membres de la diversité sexuelle par l'entremise d'activités sociales et éducatives (Lock, 2002), telles que l'inscription des coordonnées de Gai Écoute dans l'agenda scolaire, achat de livres traitant de l'homosexualité, la bisexualité et les transidentités, pose d'affiches sur les murs dans des endroits stratégiques, activités dans le cadre de la Journée internationale contre l'homophobie (Conseil permanent de la jeunesse, 2007). Au Québec, peu de données sont disponibles sur ces groupes. Une étude récente démontre que seulement 1,3\% des 224 écoles québécoises participantes possèdent une association de ce type (Conseil permanent de la jeunesse, 2007).

Les associations d'enseignants, les regroupements professionnels ou les syndicats offrent aussi du soutien au personnel scolaire sous la forme de formations ou de partage de documentation et d'outils permettant le développement des compétences (Ryan, 2003). Ainsi, la Fédération canadienne des enseignantes et enseignants (FCEE) offre à ses membres plusieurs manuels comme Seeing the Rainbow, The Gay-Straight Student Alliance Handbook ou encore Challenging Silence, Challenging Censorship (FCEE, 2007). De son côté, la Centrale des syndicats du Québec (CSQ) a produit en 2002 une vidéo intitulée Silence S.V.P., dont l'objectif est de mettre en lumière les effets destructeurs de l'homophobie en milieu scolaire (Conseil permanent de la jeunesse, 2007). Le Comité pour la diversité sexuelle de la CSQ a aussi mis en place en 2006 deux Tables nationales de lutte contre l'homophobie; une pour le réseau scolaire secondaire, l'autre pour le réseau collégial (CSQ, 2010).

\section{Les initiatives de la communauté}

Les ateliers du Groupe régional d'intervention sociale (GRIS), que l'on retrouve entre autres dans les villes de Montréal et de Québec, sont certainement ceux qui ont le plus de visibilité 
"Ces organismes font la promotion d'une "vision positive de l'homosexualité et de la bisexualité en vue de favoriser une intégration harmonieuse des personnes gaies, lesbiennes et bisexuelles dans la société "..."

"...la création

d'un environnement sécuritaire pour tous doit dépasser largement les initiatives à petite échelle et s'incarner dans une volonté sociale et politique. " au Québec. Ces organismes font la promotion d'une "vision positive de l'homosexualité et de la bisexualité en vue de favoriser une intégration harmonieuse des personnes gaies, lesbiennes et bisexuelles dans la société » (GRIS-Québec, 2006, p. 5), par le biais d'activités de sensibilisation dans les écoles secondaires pendant lesquelles de jeunes bénévoles témoignent de leur expérience comme lesbienne, gai ou bisexuel dans le but de sensibiliser et d'informer les élèves du secondaire (Grenier, 2005). Aux ÉtatsUnis, une organisation de défense des droits, The American Civil Liberties Union (ACLU), propose son aide aux organisations qui veulent mettre en place le Making School Safe Program (Middleton, 2002) qui vise à faire la promotion d'un environnement sécuritaire et à soutenir le personnel enseignant à contrer le harcèlement lié à l'orientation sexuelle.

\section{Les initiatives institutionnelles}

Pour plusieurs des acteurs impliqués dans les stratégies de lutte à l'homophobie, la création d'un environnement sécuritaire pour tous doit dépasser largement les initiatives à petite échelle et s'incarner dans une volonté sociale et politique (Conseil permanent de la jeunesse, 2007; Ryan, 2003). Quelques programmes, dont un québécois, ont été développés en ce sens. Le plus ancien, le Massachusetts Safe School Program, créé en 1993 par le Massachusetts Department of Elementary and Secondary Education (Griffin et Ouellett, 2002), commandite la mise en place de politiques contre le harcèlement et la discrimination; forme le personnel sur les questions touchant le suicide et les moments de crises personnelles des élèves; soutient la mise en place de Gay Straight Alliances dans les écoles; et fournit du soutien professionnel pour les familles d'élèves LGBT (Szalacha, 2003). Le deuxième exemple vient de l'Ontario, plus précisément du Toronto District School Board. Cette commission scolaire a instauré en 2000 une politique antihomophobie (Anti-Homophobia, Sexual, and Equity Program) qui favorise l'usage de matériel scolaire exempt de propos discriminatoires ou violents envers les membres de la diversité sexuelle et favorise l'utilisation de matériel qui reflète l'expérience de vie des LGBT. Cette politique vise le renforcement 
du partenariat avec les groupes communautaires et le soutien au développement des connaissances (Goldstein, Collins et Halder, 2008). Le dernier exemple est celui du comité de prévention organisé par la Commission scolaire Marguerite-Bourgeoys à l'ouest de l'île de Montréal. Créé à l'automne 2004, ce comité met l'accent sur l'accès aux services de proximité (psychologues, psychoéducateurs, conseillers d'orientation, etc.) pour les jeunes LGB et à la formation de ces mêmes professionnels.

\section{Évaluation des initiatives de lutte à l'homophobie à l'école}

"Peu de chercheurs jusqu'à présent ont évalué l'implantation ou les effets des initiatives de lutte à l'homophobie dans les écoles. "

"Les initiatives plus politiques telles la création de comités de lutte à l'homophobie ou la mise en place de programmes dans les commissions scolaires sont évaluées favorablement. Elles sont associées à une diminution de l'hétérosexisme, à l'augmentation de la visibilité de l'homosexualité et des conséquences de l'homophobie."
Peu de chercheurs jusqu'à présent ont évalué l'implantation ou les effets des initiatives de lutte à l'homophobie dans les écoles (Maher, et collab., 2009). On sait toutefois que le manque de formation du personnel enseignant sur le sujet, la crainte de faire la "promotion" de l'homosexualité et la pénurie des ressources humaines et financières représentent quelques-uns des obstacles à l'émergence de projets de prévention (Conseil permanent de la jeunesse, 2007; Ryan, 2003). Par contre, la mise en place de cours dédiés à la sexualité humaine enseignés par du personnel formellement attitré, la présence de groupes communautaires et la clarté des approches de lutte à l'homophobie proposées semblent être des facteurs favorisant un environnement sécuritaire pour les jeunes (Conseil permanent de la jeunesse, 2007; Richard, 2010; Ryan, 2003). Les discussions en classe, comme celles offertes par les GRIS, sont associées à un plus grand sentiment de confort des élèves hétérosexuels envers les personnes homosexuelles (Swank, et collab., 2008).

On connait un peu mieux l'impact que peuvent avoir les Gay Straight Alliances dans les écoles, étant identifiées comme des vecteurs de développement très puissants du pouvoir d'agir (Russell, et collab., 2009), où les jeunes se retrouvent dans une position de leadership par rapport aux adultes. La présence de ces groupes est associée à un plus grand sentiment de sécurité chez les étudiants (Szalacha, 2003), toutes orientations sexuelles confondues, et à une plus grande visibilité des adultes "alliés " (Walls, Kane et Hope, 2010). Les auteurs soulignent aussi la 
nécessité de développer les compétences multiculturelles en lien avec la diversité sexuelle (Graybill, et collab., 2009).

Les initiatives plus politiques telles la création de comités de lutte à l'homophobie ou la mise en place de programmes dans les commissions scolaires sont évaluées favorablement. Elles sont associées à une diminution de l'hétérosexisme, à l'augmentation de la visibilité de l'homosexualité et des conséquences de l'homophobie (Conseil permanent de la jeunesse, 2007). Les chercheurs associent les programmes de prévention de l'homophobie à une diminution du taux de suicide, de dépression, de violence homophobe et d'absentéisme (Blake, 2001, dans Ryan, 2003).

\section{Repères conceptuels}

"Malgré les

changements

législatifs..."
Les travaux des chercheurs et intervenants s'inscrivent dans un contexte où les questions liées à la diversité sexuelle et à la lutte contre l'homophobie ont obtenu récemment la reconnaissance de l'État québécois, révélée par la Politique québécoise de lutte contre l'homophobie (Gouvernement du Québec, 2009a) et la Stratégie d'action jeunesse 2009-2014 (Gouvernement du Québec, $2009 b)$. En outre, les problèmes liés à la disparité des ressources et à l'invisibilité des communautés LGBT sont décriés depuis plusieurs années par les militants et les intervenants interpelés par les questions liées à la diversité sexuelle (Julien et Lévy, 2007). Au Québec, la majorité des organisations dédiée à la diversité sexuelle est située à Montréal (59\%) ou à Québec (13\%), tandis que $29 \%$ d'entre elles sont dispersées à travers les autres régions de la province (Commission des droits de la personne et des droits de la jeunesse, 2007). La faible visibilité publique, le manque d'espaces sociaux et l'isolement représentent certaines embûches auxquelles sont confrontées les personnes de minorités sexuelles dans leur quête d'insertion sociale (Chamberland et Paquin, 2007).

Malgré les changements législatifs au Québec et au Canada mettant fin à toute discrimination légale envers les personnes homosexuelles, les couples de même sexe et les familles 
"...l'égalité sociale n'est homoparentales, l'égalité sociale n'est pas encore pleinement pas encore pleinement atteinte pour les membres de la diversité sexuelle." atteinte pour les membres de la diversité sexuelle. En effet, la discrimination basée sur l'orientation sexuelle et la nonconformité de genre demeure très présente dans les établissements scolaires et les milieux jeunesse (Chamberland, et collab., 2011). Le stress et les craintes liés au dévoilement de l'homosexualité ou de la bisexualité accentuent considérablement la vulnérabilité des personnes de minorités sexuelles (D'Augelli, 2002). Avant d'aller plus loin, il nous semble ici nécessaire de définir brièvement deux concepts clés, soit l'homophobie et l'hétérosexisme.

\section{Homophobie et hétérosexisme}

Le terme " homophobie " tel qu'utilisé par Weinberg (1972) a d'abord servi à décrire la peur pour un hétérosexuel d'être en contact avec un homosexuel, ou encore le dégoût de soi-même chez un homosexuel, autre référence à la peur ou à l'hostilité envers les homosexuels ou les comportements homosexuels. Cette peur se manifeste généralement par de la colère, du dégoût ou de la gêne envers les homosexuels, mais elle peut aussi s'exprimer par de la violence verbale ou physique (Herek, 2004). Bien que cette définition ait évolué avec les années et les travaux issus de

"..." ce ne sont plus les personnes gaies et lesbiennes qui sont un problème, mais les attitudes négatives à leur endroit »." diverses disciplines (Hudson et Rickets, 1980), elle marque alors un changement important de paradigme puisque " ce ne sont plus les personnes gaies et lesbiennes qui sont un problème, mais les attitudes négatives à leur endroit » (Bastien Charlebois, 2011, p.117). Toutefois ce terme a soulevé un nombre important de critiques en lien notamment avec les limites «psychologisantes » de cette définition et parce que les auteurs étaient d'avis qu'il occulte les racines sociales, culturelles et politiques de la discrimination (Bastien Charlebois, 2011; Herdt et van der Meer, 2003; Herek, 2004; Maher, et collab., 2009). Plus récemment, Welzer-Lang (1994) insistait sur l'aspect social de l'homophobie, de l'importance du phénomène sur les rapports sociaux en le qualifiant d'acte de pouvoir. D'autres auteurs font référence à l'homophobie comme à une "hostilité psychologique et sociale " (Conseil permanent de la jeunesse, 2007) ou une " réaction agressive de rejet » (Demczuk, et collab., 2003). 
"Les discours et les pratiques hétérosexistes créent l'illusion que tout le monde est hétérosexuel en occultant la diversité réelle des orientations sexuelles..."

"Cette forme de discrimination ne semble possible que dans "une société patriarcale où le masculin l'emporte encore sur le féminin »."

"Les discours et les pratiques hétérosexistes créent ainsi l'illusion que tout le monde est hétérosexuel en occultant la diversité réelle des orientations sexuelles."
L'hétérosexisme, pour sa part, est défini comme «la promotion de la supériorité de l'hétérosexualité comme modèle relationnel par les institutions sociales. Les discours et les pratiques hétérosexistes créent l'illusion que tout le monde est hétérosexuel en occultant la diversité réelle des orientations sexuelles [...] [et] il accorde des privilèges au groupe dominant (les hétérosexuels) (Demczuk, et collab., 2003 p. 18). L'hétérosexisme est alors une forme spécifique de sexisme, puisqu'il émerge d'une réaction négative vis-à-vis de la «transgression » des attentes sociales par les hommes et les femmes (Conseil permanent de la jeunesse, 2007; Welzer-Lang, 1994). Cette forme de discrimination ne semble possible que dans « une société patriarcale où le masculin l'emporte encore sur le féminin " (Grenier, 2005 p. 14). Herek (2004) y fait référence en tant que système qui procure le "rationnel » et la structure à l'actualisation des comportements homophobes. Comme le mentionne si bien Bastien Charlebois (2011, p.129), ce que l'hétérosexisme traduit, c'est le fait que « les injustices commises à l'endroit de personnes LGBT ne se résument pas à quelques rares débordements émotifs et gestes d'agression ". Dans un tel système, la discrimination et la violence sont considérées comme des réponses appropriées, voire nécessaires. Cette promotion de l'hétérosexualité comme modèle à suivre, et la subordination sine qua non des autres orientations sexuelles, par les institutions sociales (Neisen, 1990) est plus évidente à titre de discours s'articulant « autour de notions telles la différenciation et la complémentarité des sexes (l'amour entre un homme et une femme est plus naturel, car il conduit à la procréation) ou la normativité hétérosexuelle " (Commission des droits de la personne et des droits de la jeunesse, 2007, p. 12). Les discours et les pratiques hétérosexistes créent ainsi l'illusion que tout le monde est hétérosexuel en occultant la diversité réelle des orientations sexuelles (Herek, 2004).

\section{Objectifs de la recherche}

Cette recherche exploratoire avait comme objectif principal de documenter les pratiques émergentes visant à contrer les violences 
homophobes auprès des jeunes de minorités sexuelles dans la région de l'Outaouais. À partir des récits de onze intervenants et enseignants, cette exploration des pratiques a permis plus spécifiquement 1 . de décrire les stratégies de prévention ou de sensibilisation développées par les divers acteurs sociaux pour contrer la discrimination basée sur l'orientation sexuelle et ses manifestations; 2. d'identifier les différentes collaborations établies entre les écoles et la communauté, ainsi que les facteurs facilitant de telles alliances; 3 . d'identifier les facteurs qui favorisent l'émergence des pratiques en milieu scolaire et jeunesse et ceux qui les inhibent.

\section{Méthodologie}

Cette étude est de type descriptif et une méthode qualitative a été privilégiée. La recherche a été menée en partenariat avec le programme Jeunesse Idem $^{1}$ du Bureau régional d'action sida (BRAS). Implanté dans la région de l'Outaouais depuis plus de quinze ans, Jeunesse Idem travaille en collaboration avec les écoles secondaires de la région, les maisons de jeunes ainsi que d'autres organismes oeuvrant auprès des adolescents, ce qui a facilité de façon considérable le recrutement des répondants puisqu'il a été réalisé auprès des milieux déjà identifiés par notre partenaire. Les directeurs des écoles secondaires et les coordonnateurs des maisons de jeunes ou d'autres organismes jeunesse ont été contactés et informés des objectifs de l'étude. À la suite de ce premier contact téléphonique, un résumé du projet leur a été envoyé par courrier électronique. Ils ont été invités à identifier parmi leur équipe de travail les personnes qui ont développé des initiatives ou des activités de lutte contre la discrimination basée sur l'orientation sexuelle, et qui accepteraient de nous faire part de leur expérience. L'échantillon est donc intentionnel, c'est-à-dire qu'il est constitué de personnes qui répondent au type idéal par rapport aux objectifs de notre recherche (Ouellet et St-Jacques, 2000). Le principal critère de sélection qui a permis d'inclure un répondant ou une répondante est celui d'avoir mis ou tenté de mettre en place une 
activité visant la sensibilisation des adolescents en milieu scolaire ou jeunesse à la réalité des jeunes de minorités sexuelles, visant ainsi à contrer les violences homophobes dans leur milieu. La taille de l'échantillon a été déterminée avant tout par l'évolution de la recherche, le jugement de la chercheuse, mais aussi par le critère de saturation empirique (Pires, 1997). Des entrevues semistructurées d'une durée d'environ une heure ont été conduites auprès des répondants issus des milieux identifiés. L'utilisation de questions découlant de thèmes spécifiques a été privilégiée, permettant d'une part d'obtenir des réponses liées aux contextes et circonstances dans lesquelles les répondants développent les stratégies d'intervention et d'autre part, de faciliter l'identification des facteurs qui favorisent l'émergence des pratiques innovatrices et les défis auxquels les divers acteurs sociaux sont confrontés. De façon plus spécifique, les thèmes abordés concernaient les éléments sur l'expérience professionnelle, les clientèles de l'école ou de l'organisme, les situations ou les manifestations à caractères homophobes vécues depuis les trois dernières années, les interventions de lutte à l'homophobie structurées et celles développées de façon spontanée. Les entrevues ont été retranscrites intégralement et la technique d'analyse thématique (Mayer, et collab., 2000) a été appliquée à l'ensemble de ces verbatims.

\section{Résultats}

\section{Portrait des répondantes et des répondants}

L'échantillon est composé de onze personnes $(n=11)$, soit huit femmes et trois hommes. Ce sont tous des professionnels de l'éducation ou des services sociaux qui travaillent auprès des adolescents ou des jeunes adultes. Les répondants sont issus de professions et de formations variées : travail social (4), psychoéducation (1), éducation spécialisée (2), éducation (1), adaptation scolaire et sociale (1) et animation culturelle (2). Trois d'entre eux travaillent dans des écoles secondaires et deux sont au niveau collégial. Quatre personnes oeuvrent dans des organismes 
communautaires jeunesse et, finalement, deux intervenants sont rattachés à des établissements du réseau de la santé et des services sociaux. La majorité de nos répondants $(\mathrm{n}=9)$ travaille en milieu urbain, mais deux sont situés en région semi-urbaine. Ils ont en moyenne quatorze ans d'expérience professionnelle dans leur domaine respectif.

\section{Les pratiques de lutte contre l'homophobie : types d'initiatives et acteurs impliqués}

"Les jeunes de minorités sexuelles victimes d'homophobie se tournent le plus souvent vers leurs amis ou leur famille lorsqu'ils ont besoin de soutien."

"La plupart des intervenants et des enseignants interrogés veulent dispenser aux jeunes qu'ils côtoient dans le cadre de leur travail une éducation sexuelle appropriée et non discriminante. "
Les jeunes de minorités sexuelles victimes d'homophobie se tournent le plus souvent vers leurs amis ou leur famille lorsqu'ils ont besoin de soutien (Carnelley, et collab., 2011). Dans une moindre mesure, certains vont chercher de l'aide auprès des ressources professionnelles ou du personnel enseignant de leur école, mais leur accessibilité ou leur disponibilité fluctuent (Conseil permanent de la jeunesse, 2007). Les informations récoltées auprès des répondants ont permis d'établir que les pratiques de lutte contre l'homophobie sont créées et mises en place par différents acteurs et à plusieurs niveaux, soit les intervenants et les enseignants (parfois en collaboration avec les jeunes), la communauté et les institutions publiques et parapubliques. Par ailleurs, ces pratiques sont influencées par le niveau de connaissances et l'attitude des adultes qui oeuvrent auprès des jeunes.

\section{Les savoirs et le savoir-être des intervenants et du personnel scolaire}

La plupart des intervenants et des enseignants interrogés veulent dispenser aux jeunes qu'ils côtoient dans le cadre de leur travail une éducation sexuelle appropriée et non discriminante. Cependant, ils reconnaissent que leurs interventions demeurent sporadiques. De plus, la majorité des répondants déplorent leur manque de formation quant aux questions associées à la diversité sexuelle. En général, les répondants proposent la définition communément admise de l'homophobie, en spécifiant qu'il s'agit d'une " discrimination ", d'une "violence " ou d'un " malaise " à l'égard des personnes homosexuelles. De plus, ils illustrent leur 
définition par des exemples qui évoquent les différentes formes de violence homophobe qui surviennent dans les rapports entre jeunes (verbale, psychologique, physique). Toutefois, ils estiment que l'homophobie est plus présente chez les garçons que chez les filles et que la bisexualité est en quelque sorte une "mode " au secondaire. À ce propos, une travailleuse sociale en milieu scolaire explique:

«Moi, je vois une différence entre les gars et les filles. La violence semble plus virulente chez les garçons. On dirait que l'homosexualité vient menacer leur masculinité. [...] J'ai eu tellement de petites filles en secondaire $2 \ldots$ elles étaient toutes bisexuelles! Je me suis beaucoup questionnée, parce que je me suis dit : "Bien c'est quoi ça? Est-ce que c'est juste une mode?” Mais elles ne vivent pas d'homophobie par rapport à ça. Au contraire, c'est quasiment recherché." (Claudine) $^{2}$

Le concept d'hétérosexisme, quant à lui, est inconnu de la plupart des personnes interrogées, comme en témoigne la réponse d'une répondante : "L'hétéro... quoi? J'ai jamais entendu ça de ma vie! » Or, bien que le mot lui-même soit absent de leur vocabulaire, elles font toutefois référence à une "violence systémique " par laquelle l'hétérosexualité est imposée comme étant la norme sociale.

\section{Les pratiques individuelles et de groupe}

Certains intervenants et enseignants rencontrés ne se contentent pas d'éviter le sujet ou de suivre le curriculum scolaire fourni par le Ministère ou leur organisme; ils bonifient l'apprentissage par des visionnements de films, par des lectures obligatoires ou abordent plus précisément le thème de l'homosexualité au gré des questions des jeunes et des manifestations d'homophobie dont ils sont témoins à l'école ou dans les milieux jeunesse. D'après les répondants, la lutte contre l'homophobie exige la collaboration de plusieurs acteurs : élèves, enseignants, direction et même la 
communauté, tels les policiers et les organismes communautaires. Un répondant mentionne que dans son établissement collégial un comité composé de membres du personnel et d'élèves a vu le jour il y a quelques années. Ce comité s'assure que des projets de lutte à l'homophobie seront réalisés tout au long de l'année scolaire. Ces projets peuvent prendre diverses formes : inscription des coordonnées de Gai Écoute ${ }^{3}$ dans l'agenda scolaire, présentations d'ateliers offerts par les organismes communautaires, achat de livres traitant de l'homosexualité, pose d'affiches sur les murs et, surtout, proposition d'activités dans le cadre de la Journée internationale contre l'homophobie. La récurrence annuelle de ce comité parât toutefois incertaine puisqu'il ne dispose pas d'un local permanent et que le leadership étudiant fluctue au gré des années. À chaque rentrée scolaire, la survie du comité est donc menacée. D'autres pratiques ont été décrites par les répondants, dont la création d'un recueil de textes et l'évaluation des besoins des élèves par l'entremise d'un questionnaire.

\section{Les pratiques communautaires}

En Outaouais, les écoles et les milieux jeunesse ont l'opportunité d'accueillir un représentant de Jeunesse Idem à l'occasion d'un atelier de sensibilisation ou la tenue d'un kiosque d'information. L'objectif des ateliers est d'informer et de sensibiliser les jeunes afin de diminuer les tabous et les préjugés liés à l'homosexualité, à la bisexualité et à la transsexualité. Durant une période scolaire ou une séance d'environ 75 minutes, l'auditoire peut poser des questions au sujet des orientations sexuelles et de l'identité de genre. Ayant accepté de participer à ce projet de recherche par l'entremise d'une invitation de Jeunesse Idem, tous les répondants sont au courant des activités de l'organisme et font appel à ses services sur une base annuelle. Une psychoédactrice a d'ailleurs mis sur pied un groupe de soutien à l'intention des jeunes de minorités sexuelles en collaboration avec Jeunesse Idem.

\section{Les pratiques institutionnelles}

La dernière source de prévention de l'homophobie en milieu scolaire que les répondants ont mentionnée réfere aux 
«... il est peu probable que le phénomène de l'homophobie se résorbe si une volonté politique et sociale dépassant les initiatives des enseignants ou des groupes communautaires ne s'incarne pas dans les écoles et dans les milieux jeunesse. " pratiques institutionnelles, faisant référence principalement aux commissions scolaires, au gouvernement et à ses différents ministères. Selon sept de nos répondants, il est peu probable que le phénomène de l'homophobie se résorbe si une volonté politique et sociale dépassant les initiatives des enseignants ou des groupes communautaires ne s'incarne pas dans les écoles et dans les milieux jeunesse. À ce propos, une répondante donne comme exemple un comité de prévention organisé par une commission scolaire montréalaise. Selon les dires de cette intervenante, ce projet met l'accent sur l'accès aux services de proximité pour les jeunes de minorités sexuelles et sur la formation des intervenants et du personnel scolaire. Or, elle n'a jamais entendu parler d'une telle initiative en Outaouais.

\section{Facteurs qui favorisent l'émergence des pratiques de lutte contre l'homophobie}

"Nous devons éduquer nos enfants à être ouverts puis de respecter l'autre pour ce qu'il est..."
La description des diverses pratiques décrites par les répondants a permis subséquemment de dégager certains facteurs qui favorisent leur émergence dans les écoles et les milieux jeunesse. D’abord, la conviction qui anime les intervenants et le personnel scolaire de mettre fin aux discriminations basées sur les orientations sexuelles évoque un engagement citoyen ou une valeur personnelle qui va au-delà de leurs responsabilités professionnelles. Une enseignante du secondaire explique :

"C'est mon rôle en tant que citoyenne. Il faut faire une différence dans la vie. Il faut le faire, peu importe si tu es avocat ou maman à la maison. Nous devons éduquer nos enfants à être ouverts puis de respecter l'autre pour ce qu'il est [...] La lutte contre l'homophobie, ça doit être l'affaire de tout le monde. Pourquoi on ne mettrait pas un roman abordant l'homosexualité comme livre obligatoire dans un cours de français? " (Mireille)

L'appui de l'équipe ou de la direction est un autre facteur mentionné par les répondants qui contribue indubitablement 
selon eux à privilégier une approche visant à lutter contre les discriminations. Le sentiment de pouvoir compter sur ses collègues et de ne pas porter seul cette responsabilité procure, selon une intervenante communautaire, un "gros plus " vers l'adoption de pratiques au sein de son organisme. Elle poursuit en faisant état du climat qui règne dans son équipe de travail :

« On est pas mal toutes sur la même longueur d'onde d'après moi. On essaie toutes de faire des petits gestes et essayer de sensibiliser les jeunes par rapport à ça. Faire des activités. Ou quand on entend un commentaire, on essaie de réagir le plus rapidement possible. Tout le monde le fait, c'est comme ça. On est une équipe. C'est le “nous", ce n'est pas juste le "je”." (Véronique)

"...la présence d'un organisme régional spécialisé en diversité sexuelle assure selon les répondants une continuité dans les services auprès des jeunes..."
Outre l'ouverture au sein du milieu de travail, la présence d'un organisme régional spécialisé en diversité sexuelle assure selon les répondants une continuité dans les services auprès des jeunes, en plus d'être une référence professionnelle sur le plan des connaissances associées aux réalités LGBT et de l'intervention auprès des jeunes de minorités sexuelles et de leur famille.

Finalement, tous les répondants ont mentionné que leurs pratiques de lutte contre l'homophobie découlent d'un élément déclencheur survenu dans leur milieu. Confrontés à une situation jugée urgente qui met en lumière leur manque de préparation ou de ressources pour y répondre adéquatement à ce moment-là, les répondants ont alors dû réfléchir aux moyens à mettre place pour relever ce défi. Interrogée à cet effet, une psychoéducatrice raconte un épisode survenu dans son école secondaire :

"C'était un élève en particulier du secondaire 1 qui est venu se confier. On sentait qu'on manquait d'instrument pour l'aider. Les plus jeunes, ils ne peuvent pas nécessairement aller en ville pour des rencontres. C'est loin, il faut qu'ils prennent l'autobus. Au niveau de la confidentialité des parents, ça pose problème. 
Est-ce qu'on peut faire quelque chose à l'école qui permettrait de répondre au besoin? Il fallait qu'on fasse quelque chose. " (Chantal)

\section{Facteurs qui inhibent l'émergence de pratiques de lutte contre l'homophobie}

"Il n'y a pas de réseau entre les différents milieux scolaires, autant au niveau de l'homosexualité que des autres réalités de l'intervention.»

"...On nous dit de ne pas parler de ça devant les jeunes afin de ne pas les influencer. On ne parle pas de l'homosexualité parce que ça peut donner des idées. »...»
Outre les facteurs qui favorisent l'émergence de pratiques, les récits des répondants font état de ceux qui empêchent ou qui compromettent les initiatives de lutte contre l'homophobie. La méconnaissance des pratiques ou des programmes de prévention, associée à un certain isolement sur le plan professionnel, est l'un des éléments soulevés par les répondants. À ce propos, une travailleuse sociale en milieu scolaire explique :

« Moi, je me retrouve toujours seule. Il n'y a pas de réseau entre les différents milieux scolaires, autant au niveau de l'homosexualité que des autres réalités de l'intervention. Qu'est-ce qui se fait à telle école? Je n'en ai aucune idée. Au niveau de l'homophobie, est-ce qu'ils font quelque chose comme activité par rapport à ça? Je ne sais pas.» (Élise)

Les réactions réfractaires, négatives ou carrément homophobes des autres membres de l'équipe sont également identifiées par un des répondants comme étant un frein à la sensibilisation des jeunes aux réalités homosexuelles. L'homophobie en milieu de travail perçue par cet éducateur spécialisé en milieu hospitalier représente une embûche importante et souvent sous-estimée, selon lui. Il dénonce d'ailleurs les mythes qui entourent l'éducation sexuelle, notamment en ce qui a trait aux orientations sexuelles :

"C'est clair que, ici, on marche sur des œufs. Puis même encore dernièrement, on nous a demandé d'être beaucoup plus discret. [...] On nous dit de ne pas parler de ça devant les jeunes afin de ne pas les influencer. On ne parle pas de 
"...leur charge de

travail sans cesse croissante compromet leur disponibilité à mettre fin aux insultes à connotation homophobe." l'homosexualité parce que ça peut donner des idées. » (Richard)

En outre, les contraintes de temps et de ressources sont unanimement pointées du doigt par les répondants. Tiraillés par les exigences ministérielles et leurs responsabilités professionnelles, ils estiment que leur charge de travail sans cesse croissante compromet leur disponibilité à mettre fin aux insultes à connotation homophobe :

"Même moi quand je me promène dans l'école et que j'entends tous les mots homophobes de la planète, je pourrais reprendre le jeune :"Sais-tu qu'est-ce que tu viens de dire? Qu'est-ce que ça veut dire tapette?" [...] Je pourrais le reprendre puis faire un petit commentaire ou une intervention adéquate, mais je manque de temps...» (Claudine)

Finalement, l'absence de plainte formelle de la part des élèves sème une interrogation chez certains des répondants. Ils se demandent si l'homophobie est un problème réellement présent dans leur milieu, puisque le silence chez les jeunes à ce sujet indique selon eux une absence de problème. Toutefois, une éducatrice spécialisée au secondaire estime que les adultes ont une part de responsabilité et doivent mettre en place des mécanismes pour accueillir les plaintes, les questionnements et les préoccupations des adolescentes et des adolescents. Or, elle avoue ne pas aborder spécifiquement cette question lors de ses interventions, puisque l'homophobie est considérée comme une forme d'intimidation et est plutôt nommée comme telle et abordée sous cet angle, au même titre que le racisme. Qui plus est, elle reconnait que la lutte contre l'homophobie n'est pas une priorité dans son école et ne le deviendra vraisemblablement pas, à moins qu'un événement dramatique survienne :

"C'est parce qu'on n'a pas de problème non plus. Parce que si on avait des problèmes, on s'en 
occuperait. On réagirait comme on a fait avec le racisme. [...] C'est plate à dire, mais je pense qu'il faudrait qu'il y ait une catastrophe pour qu'on bouge, comme au Saguenay avec le petit gars disparu... » (Louise)

\section{Discussion}

\section{"Les pratiques}

décrites par les

répondants concernent

principalement des

discussions avec les

jeunes, ainsi que la création de comités de sensibilisation ou de soutien. "

"...le contact avec des personnes de minorités sexuelles est fortement associé à des attitudes d'ouverture envers les membres de la diversité sexuelle et les discussions en classe permettent ce type de contact. "
Cette étude contribue à l'avancement des connaissances sur les pratiques de lutte contre l'homophobie dans les milieux scolaires et jeunesse. Les pratiques décrites par les répondants concernent principalement des discussions avec les jeunes, ainsi que la création de comités de sensibilisation ou de soutien. Ces enseignants et intervenants sociaux souhaitent mettre fin à la violence homophobe et prendre les moyens nécessaires pour atteindre leur objectif, ce qui corrobore les résultats obtenus par Grenier (2005) et par Martinez et Phillips (2009). Les discussions en classe, comme celles offertes par Jeunesse Idem, sont associées à un plus grand sentiment de confort en compagnie de personnes homosexuelles chez les étudiantes et étudiants hétérosexuels (Swank, et collab., 2008). Il est impossible toutefois de savoir si les discussions sont la cause de ce sentiment de confort ou si celui-là est causé par un environnement scolaire ouvert dans lequel s'intègre ce genre d'activité préventive. Or, le contact avec des personnes de minorités sexuelles est fortement associé à des attitudes d'ouverture envers les membres de la diversité sexuelle et les discussions en classe permettent ce type de contact (Grenier, 2005). Les deux tiers des écoles québécoises interrogées par le Conseil permanent de la jeunesse (2007) font appel à une ressource externe en matière de lutte contre l'homophobie, mais ces activités ont lieu de manière sporadique dans la majorité des cas. Les initiatives de ces organismes ne représentent pas une panacée, car leur champ d'action est restreint aux milieux urbains et leurs alentours et ils dépendent en grande partie de ressources humaines bénévoles (Chouinard, 2011). 
"...l'absence de plainte de la part des jeunes fait en sorte que la lutte contre l'homophobie n'est pas jugée comme étant une priorité au sein de certains établissements..."
Certains facteurs inhibent l'émergence des pratiques de lutte contre l'homophobie, dont l'isolement et l'absence de réseautage professionnel, l'ignorance et les préjugés de certains membres du personnel ainsi que la pénurie des ressources humaines et financières. La crainte de faire la "promotion " de l'homosexualité représente quant à elle un autre obstacle aussi identifié par le Conseil permanent de la jeunesse (2007). Selon Ryan (2003), les écoles de formation professionnelle et les universités sont réticentes à aborder les questions liées aux orientations sexuelles et à l'identité de genre. Conséquemment, les futurs enseignants et intervenants sociaux n'ont pas l'occasion de se (re)questionner sur leurs perceptions à l'égard des réalités LGBT, ni de cerner leurs malaises et leurs préjugés. Cette méconnaissance fait en sorte que la plupart d'entre eux entretiennent la conviction que les personnes de minorités sexuelles ne vivent aucune difficulté particulière, ce qui constitue une barrière importante dans l'expression de leurs besoins. Par ailleurs, l'absence de plainte de la part des jeunes fait en sorte que la lutte contre l'homophobie n'est pas jugée comme étant une priorité au sein de certains établissements scolaires et organismes jeunesse. Or, l'étude de Chamberland et collab. (2011) révèle que $27,1 \%$ des victimes d'un incident homophobe n'ont pas dénoncé la situation aux adultes responsables parce qu'elles avaient l'impression que rien ne sera fait pour corriger la situation.

\section{Vers l'instauration de milieux sécuritaires pour les jeunes}

La prévention de l'homophobie ne se cantonne pas seulement aux discussions en classe à propos des orientations sexuelles ou à la mise en place d'un comité pour la diversité sexuelle dans un milieu jeunesse. Griffin et Ouellett (2002) estiment que des

"L'action individuelle n'est qu'une " pointe " du triangle, les deux autres étant les préjugés véhiculés au sein de la société et les normes formelles et informelles des institutions. » politiques claires, tant au niveau local que provincial, soutenues par des ressources techniques, légales et financières, et conjuguées avec l'implication de l'ensemble de la communauté scolaire (administrateurs, enseignants, intervenants communautaires et élèves) sont primordiales pour la création d'un environnement sécuritaire pour les jeunes. À ce propos, le modèle triangulaire de l'oppression de McCaskell (2005) met en lumière un écueil dans une démarche pour en finir avec les oppressions, soit celui de 
"...la « pédagogie

féministe est particulièrement efficace pour contrecarrer la discrimination parce qu'elle prend les élèves où ils sont, valorise leur expérience..." focaliser uniquement sur les comportements individuels. L'action individuelle n'est qu'une "pointe " du triangle, les deux autres étant les préjugés véhiculés au sein de la société et les normes formelles et informelles des institutions. Les manifestations d'homophobie telle la violence verbale ne sont alors que la pointe de l'iceberg (Goldstein, et collab., 2008). La prévention doit donc s'attaquer tant aux politiques scolaires internes qu'à la transformation des perceptions sociales (Lipkin, 2004). Le terme "hétérosexisme» (culturel, social et politique) se distingue alors de celui d'" homophobie " (comportemental, individuel), puisqu'éradiquer l'hétérosexisme est plus prometteur à long terme que de réprimer les manifestations homophobes (Bastien Charlebois, 2011; Swank, et collab., 2008). Bien qu'aborder le problème de l'homophobie sous l'angle de la lutte à la discrimination pour sensibiliser les adultes à l'importance de la prévention en général soit utile (Schniedewind et Cathers, 2003), il est plus efficace d'énoncer clairement des politiques contre l'homophobie et l'hétérosexisme pour mettre fin à la violence et à l'intimidation (Walton, 2004).

\section{Vers une pédagogie expérientielle}

Une pédagogie utilisant les principes féministes de validation de l'expérience personnelle permet aux jeunes de définir euxmêmes leur réalité affective et sexuelle. À ce propos, Crumpacker et Vander Haegen (1993, p. 102 [notre traduction]) estiment que la "pédagogie féministe est particulièrement efficace pour contrecarrer la discrimination parce qu'elle prend les élèves où ils sont, valorise leur expérience, et, à partir de là, élargit les définitions et ébranle les façons étroites de voir la réalité ». Offrir l'occasion aux personnes - et en ce qui nous concerne, les jeunes - de minorités sexuelles de s'exprimer et de relater leurs expériences de l'oppression, mais aussi de leur épanouissement (grâce à des conférences, à des lectures, à des visionnements de films, à des témoignages, etc.) augmente leur estime de soi et leur pouvoir d'agir (Lipkin, 2004). Cette approche d'intervention peut aussi mettre en relief la socialisation des garçons inspirée par les stéréotypes d'une masculinité axée sur la violence, le sexisme et le 
rejet du féminin (McCann, Minichiello et Plummer, 2009). Selon Lock (2002), l'enseignement de la tolérance (teaching tolerance) débute par la règle du "zéro indifférence ", ce qui signifie qu'il faut intervenir activement devant les manifestations de harcèlement, sans viser uniquement la confrontation ou la punition. Ainsi, la violence ne peut être excusée en la banalisant ou en invoquant la «nature » bagarreuse des garçons (Tharinger, 2008).

\section{Conclusion}

«...il est tout à fait possible d'adopter des pratiques qui découlent des savoirs scientifiques, c'est à dire qui évitent la confrontation, ébranlent les stéréotypes liés aux genres et soulignent l'impact de la discrimination sur toutes les personnes, et ce, peu importe leur orientation sexuelle ou leur âge."
L'étude qualitative dont il été question dans cet article propose un portrait descriptif des pratiques de lutte contre l'homophobie dans les milieux scolaires et jeunesse de l'Outaouais, en identifiant notamment les facteurs qui favorisent leur émergence et ceux qui les inhibent. Il s'en dégage la nécessité de créer des alliances afin de proposer une réponse systémique au phénomène de l'homophobie et de l'hétérosexisme chez les jeunes. Avec un peu de flexibilité, il est tout à fait possible d'adopter des pratiques qui découlent des savoirs scientifiques, c'est à dire qui évitent la confrontation, ébranlent les stéréotypes liés aux genres et soulignent l'impact de la discrimination sur toutes les personnes, et ce, peu importe leur orientation sexuelle ou leur âge. Le défi se trouve donc surtout dans la systématisation de la prévention et la concertation des acteurs de tous les niveaux. Lorsque ce défi sera relevé, les pratiques innovatrices pourront alors non seulement émerger, mais s'implanter à long terme dans les milieux scolaires et jeunesse.

\section{Notes}

Le programme Jeunesse Idem est le seul service d'information et de soutien pour les jeunes de minorités sexuelles de 14 à 25 ans en Outaouais. Il a pour mandat de briser l'isolement des jeunes en leur permettant de partager avec leurs pairs sans être jugés. Les objectifs du programme sont : 1. fournir des modèles positifs de vécu gai, lesbien, bisexuel, transgenre et transsexuel; 2 . favoriser l'évolution personnelle, les relations interpersonnelles et le sentiment d'appartenance des jeunes LGBT de la région et 3. faire la promotion de la santé. 
2 Tous les noms sont fictifs.

3 Gai Écoute est un centre d'aide et de renseignements à l'intention des personnes interpellées par les questions liées de près ou loin aux orientations sexuelles. Les services d'écoute téléphonique et de clavardage sont accessibles sans frais à travers le Québec.

\section{Bibliographie}

ADAM, Barry D. (1998). "Theorizing homophobia », Sexualities,Vol. 1, No 4, p. 387-404.

BASTIEN CHARLEBOIS, Janik (2010). " "L'homophobie naturelle” des garçons adolescents : essor et ressorts d'explications déterministes ", Cahiers de recherche sociologique, № 49, p. 181-201.

BASTIEN CHARLEBOIS, Janik (2011). "Au-delà de la phobie de l'homo : quand le concept d'homophobie porte ombrage à la lutte contre l'hétérosexisme et l'hétéronormativité ", Reflets, revue d'intervention sociale et communautaire, $\mathrm{Vol} .17, \mathrm{~N}^{\circ}$ 1, printemps, p. 112-149.

BAUERMEISTER, José A., Michelle Marie JOHNS, Theo G.M. SANDFORT, Anna EISENBER G, Arnold H. GROSSMAN et Anthony R. D’Augelli (2010). "Relationship trajectories and psychological well-being among sexual minority youth ", Journal of youth adolescence, $\mathrm{N}^{\circ}$ 39, p. 1148-1163.

BEEMYN, Brett Genny (2005). « Making campuses more inclusive of transgender students ", Journal of gay \& lesbian issues in education, Vol. 3, No 1, p. 77-87.

BEEMYN, Brett Genny, Andrea DOMINGUE, Jessica PETTITT et Todd SMITH (2005). «Suggested steps to make campuses more trans-inclusive ", Journal of gay $\&$ lesbian issues in education, Vol. 3, N 1, p. 89-94.

BENTON, Jeremy (2003). "Making schools safer and healthier for lesbian, gay, bisexual and questioning students ", The Journal of school nursing,Vol. 19, № 5, p. 251-259.

BUSTON, Katie, et Graham Hart (2001). "Heterosexism and homophobia in Scottish school sex education: Exploring the nature of the problem ", Journal of adolescence, $\mathrm{N}^{\circ}$ 24, p. 95-109.

CARNELLEY, Katherine B., Erica G. HEPPER, Colin HICKS et William TURNER (2011). "Perceived parental reactions to coming out, attachment, and romantic relationship views ", Attachment and human development, Vol. 13, № 3, p. 217-236

CENTRALE DES SYNDICATS DU QUÉBEC (2010) «Rapport d'activités 2006-2009 du Comité pour la diversité sexuelle ", réf. du 14 octobre 2010, http://www.csq.qc.net

CHAMBERLAND, Line, Gilbert ÉMOND, Danielle JULIEN, Joanne OTIS et Bill RYAN (2010). "L'homophobie en milieu scolaire au Québec : résultats d'une enquête sur les perceptions des élèves de niveau secondaire ", [Présentation PowerPoint], Conférence "Comprendre et intervenir dans le respect de la diversité sexuelle ", Québec, Québec.

CHAMBERLAND, Line, Gilbert ÉMOND, Michäl BERNIER, Gabrielle RICHARD, MariePier PETIT, Marilyne CHEVRIER, Bill RYAN, Joanne OTIS et Danielle JULIEN (2011). L'homophobie à l'école secondaire au Québec. Portrait de la situation, impacts et pistes de solution, Rapport de recherche, Montréal, Université du Québec à Montréal, 33 p.

CHAMBERLAND, Line, Blye W. FRANK et Jacine RISTOCK (2009). Diversité sexuelle et constructions de genre, Coll. "Santé et Société ", Québec, Presses de l'Université du Québec, 409 p.

CHAMBERS, Deborah, Joost VAN LOON et Estella TINCKNELL (2004). "Teacher's views of Teenage sexual morality ", British journal of sociology of education, Vol. 25, No 5, p. 563-576. 
CHOUINARD,Vincent (2010). «La prévention de l'homophobie et de l'hétérosexisme à l'école : exemple au collégial ", Service social,Vol. 56, No 1, p. 43-53.

CHOUINARD,Vincent (2011). La prévention de l'homophobie et de l'hétérosexisme à l'école secondaire : besoins et perceptions des enseignantes et des enseignants, Mémoire de maîtrise, Québec, Université Laval, 158 p.

COMMISSION DES DROITS DE LA PERSONNE ET DES DROITS DE LA JEUNESSE (2007). De l'égalité juridique à l'égalité sociale : vers une stratégie nationale de lutte contre l'homophobie, Rapport du groupe de travail mixte contre l'homophobie, Québec, 108 p.

CONSEIL PERMANENT DE LA JEUNESSE (2007). Sortons l'homophobie du placard... et de nos écoles secondaires, Québec, Gouvernement du Québec, 136 p.

CRUMPACKER, Laurie, et Eleanor M.VANDER HAEGEN (1993). « Pedagogy and prejudice: Strategies for confronting homophobia in the classroom ", Women's studies quarterly, Vol. 21, $\mathrm{N}^{\circ}$ 3/4, p. 94-106.

D'AUGELLI,Anthony R. (2002). «Mental health problems among lesbian, gay and bisexual youths age 14 to 21 ", Clinical child psychology and psychiatry, No 7, p. 439-462.

DALEY, Andrea, Steven SOLOMON, Peter A. NEWMAN et Faye MISHNA (2007). «Traversing the margins: Intersectionalities in the bullying of lesbian, gay, bisexual and transgender youth ", Journal of gay \& lesbian social services, Vol. 19, No 3, p. 9-29.

DEMCZUK, Irène, et collab. (2003). Pour une nouvelle vision de l'homosexualité : intervenir dans le respect de la diversité des orientations sexuelles, Québec, Gouvernement du Québec.

DORAIS, Michel, et Simon-Louis LAJEUNESSE (2000). Mort ou fif: Contextes et mobiles de tentatives de suicide chez les adolescents et jeunes hommes homosexuels ou identifiés comme tels, Québec, Centre de recherche sur les services communautaires, $105 \mathrm{p}$.

DOUGLAS, Nicola, Ian WARWICK, Sophie KEMP et Geoff WHITTY (1997). Playing it safe: Responses of secondary school teachers to lesbian, gay and bisexual pupils, bullying, HIV and AIDS, Education and Section 28, London Institute of Education, $70 \mathrm{p}$.

ELZE, Diane E. (2002). «Risk factors for internalizing and externalizing problems among gay, lesbian, and bisexual adolescents ", Social work research, $\mathrm{N}^{\circ} 26$, p. 89-99.

ÉMOND, Gilbert, et Janik BASTIEN CHARLEBOIS (2007). L'homophobie, pas dans ma cour!, Rapport de recherche, Montréal, GRIS-Montréal, $150 \mathrm{p}$.

ÉMOND, Gilbert (2009). “"Tous les garçons et les filles de mon âge...” Attitudes, homophobie et tyrannie relative à l'homosexualité chez les adolescents des deux sexes dans les écoles ", dans Line Chamberland (dir.), Diversité sexuelle et constructions de genre, Coll. "Santé et Société ", Québec, Presses de l'Université du Québec, p. 19-48.

EPSTEIN, Debbie, et Richard JOHNSON (1994). Challenging lesbian and gay inequalities in education, Buckingham, Open University Press, $256 \mathrm{p}$.

FÉDÉRATION CANADIENNE DES ENSEIGNANTES ET ENSEIGNANTS (2007). « Le 17 mai, Journée nationale de lutte contre l'homophobie : un nouvel outil pédagogique vise à favoriser une meilleure compréhension des élèves BBGLT ", réf. du 14 octobre 2010, http:// www.ctf-fce.ca/Newsroom/, onglet Communiqués 2007.

GALLIHER, ReneeV., Sharon S. ROSOTSKY et Hannah K. HUGUES (2004). « School belonging, self-esteem, and depressive symptoms in adolescents: An examination of sex, sexual attraction status, and urbanicity ", Journal of youth and adolescence, $\mathrm{N}^{\circ} 33$, p. 235-245. 
GAY-STRAIGHT ALLIANCE NETWORK/ TIDES CENTER / TRANSGENDER LAW CENTER / NATIONAL CENTER FOR LESBIAN RIGHTS (2004). Beyond the binary: A tool kit gender Identity activism in schools, San Francisco, CA, Gay-Straight Alliance Network, http://www.nclrights.org/site/DocServer/beyond_the_binary.pdf

GOLDSTEIN,Tara,Anthony COLLINS et Michael HALDER (2008). «Anti-homophobia education in public schooling: A Canadian case study of policy implementation ", Journal of gay E lesbian social services, Vol. $19, \mathrm{~N}^{\circ} 3$, p. 47-66.

GOUVERNEMENT DU QUÉBEC (2009a). Politique québécoise de lutte contre l'homophobie, Québec, ministère de la Justice, $44 \mathrm{p}$.

GOUVERNEMENT DU QUÉBEC (2009b). Stratégie d'action jeunesse 2009-2014, Québec, Secrétariat à la jeunesse.

GOUVERNEMENT DU QUÉBEC (2007). Le renouveau pédagogique, réf. du 2 octobre 2010, http:// www.mels.gouv.qc.ca/lancement/Renouveau_ped/.

GRAYBILL, Emily C., Kris VARJAS, Joel MEYERS et Lauren B. WATSON (2009). «Contentspecific strategies to advocate for lesbian, gay, bisexual, and transgender youth: An exploratory study ", School psychology review,Vol. 38, No 4, p. 570-584.

GRENIER, Alain (2005). Jeunes, homosexualité et écoles : enquête exploratoire sur l'homophobie dans les milieux jeunesse de Québec, Rapport synthèse, Québec, GRIS-Québec, 13 p.

GRIFFIN, Pat, et Mathew L. OUELLETT (2002). « Going beyond gay-straight alliances to make schools safe for lesbian, gay, bisexual, and transgender students ", The policy journal of the Institute for gay and lesbian strategic studies, Vol. 6, No 1, p. 1-8.

GRIS-QUÉBEC (2006). La démystification de l'homosexualité ça commence maintenant! Guide du démystificateur automne 2006, Document inédit, Québec, QC.

HEREK, Gregory M. (1988). "Heterosexuals' attitudes toward lesbians and gay men: Correlates and gender differences ", The Journal of sex research, $\mathrm{N}^{\circ} 25, \mathrm{p} .451-477$.

HEREK, Gregory M. (2004). «Beyond “homophobia”:Thinking about sexual prejudice and stigma in the twenty-first century ", Sexuality research and social policy, Vol. 1, No 2, p. 6-24.

HOMOPHOBIE.ORG (2010). Un geste à l'école, réf. du 18 octobre 2010, http://www.homophobie.org.

KIMMEL, Michael. S., et Matthew Mahler (2003). "Adolescent masculinity, homophobia, and violence: Random school shootings 1998-2001 ", American behavioral scientist, Vol. 46, № 10, p. 1439-1458.

KITZINGER, Celia (1995). "Speaking of oppression: Psychology, politics, and the language of power ", dans E. D. Rothblum, et L.A. Bond (dirs.), Preventing heterosexism and homophobia, Thousand Oaks, CA, SAGE Publications, p. 3-19.

KOSCIW, Joseph G., et collab. (2010). The 2009 National School Climate Survey: The experiences of lesbian, gay, bisexual and transgender youth in our Nation's schools, New York, GLSEN, 24 p.

LEE, Camille (2002). "The Impact of Belonging to a High School Gay/Straight Alliance ", The high school journal, Vol. 85, No 3, p. 13-26.

LIPKIN, Arthur (2004). Beyond diversity day: A Q and A on gay and lesbian issues in schools, Lanham, Maryland, Rowman \& Littlefield Publishers, $272 \mathrm{p}$. 
LOCK, James (2002). «Violence and sexual minority youth ", Journal of school violence, Vol. 1, № 3, p. 77-89.

MAC AN GHAILL, Mairtin (1994). The making of men, Buckingham, Open University Press, 209 p. MAHER, Michael, Kimberly LANDINI, Dennis M. EMANO, Andrew M. KNIGHT, Geoffrey D. LANTZ, Michael PARRIE, Shaun PICHLER \& Linda M. SEVER (2009). " Hirschfeld to hooker to herek to high schools: A study of the history and development of GLBT empirical research, institutional policies, and the relationship between the two ", Journal of homosexuality, Vol. 56, $\mathrm{N}^{\circ}$ 7, p. 921-958.

MARTINEZ,Andrea, et Karl PHILLIPS (2009). « La promotion de l'équité en éducation sexuelle dans les écoles secondaires de la région d'Ottawa », Canadian journal of education, Vol. 32, № 1, p. 60-86.

MAYER, Robert, Francine OUELLET, Marie-Christine SAINT-JACQUES, DanielTURCOTTE et collab. (dirs.) (2000). Méthodes de recherche en intervention sociale, Boucherville, Gaëtan Morin Éditeur, $410 \mathrm{p}$.

McCANN, Pol D.,Victor MINICHIELLO et David PLUMMER (2009). «Is homophobia inevitable? Evidence that explores the constructed nature of homophobia, and the techniques through which men unlearn it ", Journal of sociology, Vol. 45, No 2, p. 201-220.

McCASKELL, Tim (2005). Race to equity: Disrupting educational inequality, Toronto, Between the Lines, $350 \mathrm{p}$.

MIDDLETON, Jennifer (2002). Making schools safe. Anti-harassment training Program, New York, American Civil Liberties Union, Lesbian and Gay Rights Project, 99 p.

NAGOSHI, Julie L., Heather K.TERRELL, Eric D. HILL, Stephanie BRZUZY, CraigT. NAGOSHI (2008). "Gender differences in correlates of homophobia and transphobia ", Sex roles, Vol. 59, $\mathrm{N}^{\circ} 7-8$, p. 521-531.

NAYAK, Anoop, et Mary Jane Kehily (1996). "Playing it straight: masculinities, homophobia and schooling ", Journal of gender studies, Vol. 5, No 2, p. 211-29.

NESMITH, Andrea A., Laura Burton et T.J. Cosgrove (1999). "Gay, lesbian, and bisexual youth and young adults ", Journal of homosexuality, Vol. 37, $\mathrm{N}^{\circ} 1$, p. 95-108.

OSBORNE, Danny, et William E. WAGNER III (2007). "Exploring the relationship between homophobia and participation In core sports among high school students ", Sociological perspectives, $\mathrm{N}^{\circ}$ 50, p. 597-613.

OUELLET, Francine, et Marie-Christine SAINT-JACQUES (2000). "Les techniques d'échantillonnage ", dans Robert Mayer, et collab. (dirs.), Méthodes de recherche en intervention sociale Montréal, Gaëtan Morin Éditeur, p. 71-89.

PIRES, Alvaro P. (1997). "Échantillonnage et recherche qualitative : essai théorique et méthodologique » dans Jean Poupart, et collab. (dirs.), La recherche qualitative : enjeux épistémologiques et méthodologiques, Montréal, Gaëtan Morin Éditeur, p. 173-206.

POTEAT, Paul, et Dorothy L. ESPELAGE (2005). « Exploring the relation between bullying and homophobic verbal content: The homophobic content agent target (HCAT) scale ", Violence and victims, $\mathrm{N}^{\circ} 20$, p. 513-528.

POTEAT, Paul (2007). "Peer group socialization of homophobic attitudes and behavior during adolescence », Child development, № 78, p. 1830-1842. 
RICHARD, Gabrielle (2010). L'éducation " aux orientations sexuelles » : représentations de l'homosexualité dans les curricula formel et informel de l'école secondaire québécoise, Mémoire de maitrise, Montréal, Université du Québec à Montréal, 184 p.

RICHARD, Gabrielle, Line CHAMBERLAND et Marie-Pierre PETIT (2013). "L'inclusion de la diversité sexuelle à l'école : les enjeux pour les élèves lesbiennes, gais, bisexuels et en questionnement ", Canadian journal of education, Vol. 36, № 1, p. 375-404.

ROPER, Emily, et Elizabeth Halloran (2007). «Attitudes toward gay men and lesbians among heterosexual male and female student-athletes ", Sex roles, $N^{\circ}$ 5, p. 919-928.

ROSE, Hillary A. (2009). "A contextual model of victimization of sexual-minority youth ", dans Line Chamberland (dir.), Diversité sexuelle et constructions de genre Diversité sexuelle et constructions de genre, Coll. "Santé et Société », Québec, Presses de l’Université du Québec, p. 75-101.

RUSSELL, Stephen T., et collab. (2009). "Youth empowerment and high school gay-straight alliances ", JOURNAL OFYOUTH ADOLESCENCE, N 38, p. 891-903.

RYAN, Bill (2003). Nouveau regard sur l'homophobie et l'hétérosexisme au Canada, Ottawa, Société canadienne du sida, $102 \mathrm{p}$.

SAEWYC, Elizabeth M. (2011). «Research on adolescent sexual orientation: Development, health disparities, stigma, and resilience ", Journal of research on adolescence, Vol. 21, № 1, p. 256-272.

SCHNIEDEWIND, Nancy, et Karen Cathers (2003). «Becoming allies for each other: An inclusive approach for confronting heterosexism in schools ", Equity E excellence in education, Vol. 36, $\mathrm{N}^{\circ}$ 2, p. 184-193.

SMITH, George W. (1998). "The ideology of "fag": The school experience of gay students », The sociological quarterly, Vol. 39, No 2, p. 309-335.

SWANK, Eric, Lisa RAIZ, Cynthia FAULKNER, Samuel FAULKNER et Latonya HESTERBERG (2008). "Comfort with gays and lesbians after a class discussion on homophobia ", American journal of sexuality education, Vol. 3, No 3, p. 255-276.

SZALACHA, Laura A. (2003). "Safer sexual diversity climates: Lessons lLearned from an evaluation of Massachusetts safe schools program for gay and lesbian students ", American journal of education, Vol. 110, No 1, p. 58-88.

TAYLOR, Catherine, et Tracey PETER (2010). Every class in every school: The first national climate survey on homophobia in Canadian schools, Final report, Toronto, Egale Canada, $152 \mathrm{p}$.

THARINGER, Deborah J. (2008). " Maintaining the hegemonic mMasculinity through selective attachment, homophobia, and gay-baiting in schools: Challenges to intervention ", School psychologie review, $\mathrm{N}^{\circ} 37$, p. 221-227.

THIBAULT, Sylvie,Vincent Chouinard et Kévin Lavoie (à paraitre). Les réponses sociales au phénomène de l'homophobie en milieu scolaire, Recension des écrits, Département de travail social, Université du Québec en Outaouais.

VALENTI, Maria, et Rebecca CAMPBELl (2009). "Working with youth on LGBT issues: Why gay-straight alliance advisors become involved ", Journal of community psychology, Vol. 37, $\mathrm{N}^{\circ} 2$, p. 228-248.

WALLS, Eugene, Sarah B. KANE et Hope WISNESKI (2010). « Gay-straight alliances and school experiences of sexual minority youth ", Youth society, Vol. 41, No 3, p. 307-332.

WALTON, Gerald (2004). " Bullying and homophobia in Canadian schools ", Journal of gay E lesbian issues in education, Vol. 1, $\mathrm{N}^{\circ}$ 4, p. 23-36. 